\title{
Reaching spatial or networking saturation in VANET
}

Serkan Öztürk*, Jelena Mišić ${ }^{1}$ and Vojislav B Mišić ${ }^{2}$

\begin{abstract}
In this article, we investigate the network transition between non-saturation and saturation regimes for a Vehicular Ad hoc Network (VANET) which is composed of mobile nodes. We combine vehicular traffic theory, queuing model, and Markov chain to evaluate the performance of the network under spatial or networking saturation for multiple data classes over control channel and service channel. Our results indicate that the vehicle density growth can result in saturation of wireless medium around the roadside unit (RSU), further resulting in buffer overflows at on board units (OBUs). We also investigate the network saturation points for different transmission ranges of a RSU. Our results show that RSU's transmission coverage has to be chosen with respect to data patterns of OBUs, minimal distance between vehicles, and number of lanes in order to avoid network saturation condition.
\end{abstract}

Keywords: IEEE 802.11p, vehicular traffic theory, free-flow regime, network saturation

\section{Introduction}

Vehicular Ad hoc Network (VANET) is a special type of Mobile Ad hoc Network (MANET) based on short-range communications among moving vehicles and between vehicles and roadside units (RSUs). IEEE 802.11p is referred to as dedicated short-range communications (DSRC) standard for wireless access in vehicular environment (WAVE). For DSRC, $75 \mathrm{MHz}$ of licensed spectrum at $5.9 \mathrm{GHz}$ has been allocated. This $75 \mathrm{MHz}$ band is divided into one central control channel $(\mathrm{CCH})$ and six service channels (SCHs) as shown in Figure 1. $\mathrm{CCH}$ is dedicated for transmission of traffic safety messages, while $\mathrm{SCHs}$ are dedicated to transfer of various application data. Both $\mathrm{CCH}$ and $\mathrm{SCH}$ support four data classes with aggressively differentiated priorities as shown in Tables 1 and 2. Each data class has its own MAC resources.

With IEEE 802.11 technologies, often a single shared wireless channel is used for both uplink (from vehicles to the RSU) and downlink (from the RSU to vehicles). Because of the distributed nature of contention, the capacity actually depends on the behavior of contending vehicles [1]. The number of contending vehicles covered by an RSU depends on the vehicle mobility and density. Furthermore, as shown in Figure 2, vehicles have different payload

\footnotetext{
* Correspondence: serkan@erciyes.edu.tr

${ }^{1}$ Erciyes University, Kayseri, Turkey

Full list of author information is available at the end of the article
}

transmission rates according to their distance to the RSU [2]. The sojourn time of a vehicle for each of the different ranges is dependent on its speed.

A VANET is unstable when a queue of any on board unit (OBU) in the network is saturated. A queue is saturated if it always has at least one frame waiting to be served. VANET cannot operate under saturation conditions because the OBU's buffer will overflow and frames queuing delay will grow unacceptably. Since all the data classes need to operate in stable conditions, the network performance must be investigated under non-saturation regime. However, network performance in non-saturation regime has received much less attention because of its complexity. In [3], the authors investigate the performance of an IEEE 802.11p-based network in non-saturation regime with static nodes.

On the other hand, spatial saturation occurs when the distance between vehicles reaches minimal (jamming) value because of the vehicular traffic congestion. While spatial saturation of vehicles during rush hours or accidents cannot be avoided, networking saturation can be avoided by proper dimensioning of resources.

In this study, we combine vehicular traffic theory, M/G/1 queuing analysis, and Markov chain analysis in order to investigate the transition between non-saturation and saturation regimes for an IEEE 802.11p-based network which is composed of mobile nodes with multiple data 


\begin{tabular}{|c|c|c|c|c|c|c|c|c|}
\hline & \multicolumn{2}{|c|}{$\begin{array}{c}\text { Critical Safety } \\
\text { of Life }\end{array}$} & \multicolumn{3}{|c|}{$\begin{array}{l}\text { Control } \\
\text { Channel }\end{array}$} & \multicolumn{3}{|c|}{$\begin{array}{l}\text { High Power } \\
\text { Public Safety }\end{array}$} \\
\hline & Ch. 172 & Ch. 174 & Ch. 176 & Ch. 178 & Ch. 180 & Ch. 182 & Ch. 184 & \\
\hline \multirow[t]{2}{*}{$\begin{array}{l}\text { 이 } \\
\infty \\
\infty \\
1\end{array}$} & $\begin{array}{l}8 \\
\varnothing \\
\infty \\
1\end{array}$ & $\begin{array}{l}0 \\
\infty \\
10 \\
10\end{array}$ & $\begin{array}{l}8 \\
\infty \\
\infty \\
1 \\
1\end{array}$ & $\begin{array}{l}8 \\
\infty \\
10\end{array}$ & $\begin{array}{l}8 \\
\text { ه } \\
\text { ம் }\end{array}$ & $\begin{array}{l}\text { 을 } \\
\text { ద் }\end{array}$ & 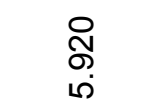 & $\begin{array}{l}\text { frequency } \\
\qquad(\mathrm{GHz})\end{array}$ \\
\hline & 'E channels. & \multicolumn{3}{|c|}{ Service Channels } & \multicolumn{3}{|c|}{ Service Channels } & \\
\hline
\end{tabular}

combinations and multiple data classes per combination. We consider the neighbourhood of a single RSU operating in non-saturation regime deployed on a bidirectional road segment. The number of vehicles in each direction (lane) under free-flow model [4] is considered as a Poisson distribution. Assuming error-prone channel conditions, we derive probability distributions for frame backoff time, waiting time in queue, collision probability of a transmission, and normalized throughput for each channel and each data class with different transmission rates depending on the vehicle's distance from the RSU.

The remainder of the article is organized as follows: in Section 2 we discuss related work and in Section 3 we develop analytical model. Section 4 presents the numerical results. Finally, Section 5 concludes the article.

\section{Related work}

Vehicular traffic flow models are classified as "microscopic", "macroscopic", and "mesoscopic" [5]. Microscopic traffic flow models describe each vehicle separately. In macroscopic models, all individual vehicles are aggregated and described as flows. The speed-flow-density relationships are used in these models $[4,6]$. Mesoscopic models combine microscopic and macroscopic elements in a unified approach. In [7] the authors investigate the connectivity of VANETs operating in free-flow regime. They use a common model [4] in vehicular traffic theory in which any observer in space sees cars passing it that are separated by exponentially distributed times.

Current state of the art in this area is a combination of saturated IEEE 802.11 model with free-flow vehicular traffic regime and spatial Poisson arrangement of vehicles

Table 1 EDCA parameter set used on the CCH (adapted from [13])

\begin{tabular}{ccccc}
\hline ACl & AC & CWmin & CWmax & AIFSN \\
\hline 1 & Background & 15 & 511 & 9 \\
0 & Best effort & 7 & 15 & 6 \\
2 & Video & 3 & 7 & 3 \\
3 & Voice & 3 & 7 & 2 \\
\hline
\end{tabular}

[1,8-12]. In [1], authors have developed an analytical framework to evaluate the upload performance for Drivethru Internet as a function of vehicle density. In [8], authors have derived an analytical model to quantify the impact of parameters such as road traffic density and vehicle speed on the download performance of moving vehicles in Drive-thru Internet systems. Authors in [9] have considered heterogeneous vehicular environments where vehicles may have different mobility characteristics. A model to estimate the collision probability in VANETs has been proposed in [10]. This model integrated the characteristics of VANETs (vehicle density and speed) into the traditional collision probability model. In [11], authors have proposed a model to improve the efficiency of communication between vehicles and RSUs. In this model, every vehicle can individually calculate its own priority of communication based on its speed and location. Authors in [12] have proposed an analytical model to evaluate the MAC throughput under different node speeds in Drivethru Internet system. All the proposed models have deployed IEEE $802.11 \mathrm{~b}$ as the wireless communication standard for VANETs instead of IEEE 802.11p. None of the proposed models have considered non-saturation regime, so far.

\section{Analytical model}

Let us consider the neighbourhood of a single RSU operating in non-saturation regime deployed on a bidirectional road segment as shown in Figure 3. According to the location to RSU, the road segment is divided into multiple regions [2]. In each region $\left(R g_{i}\right)$ within the RSU coverage area vehicles have different pay-load

Table 2 EDCA parameter set used on the SCH (adapted from [13])

\begin{tabular}{ccccc}
\hline ACl & AC & CWmin & CWmax & AIFSN \\
\hline 1 & Background & 15 & 511 & 7 \\
0 & Best effort & 15 & 511 & 3 \\
2 & Video & 7 & 15 & 2 \\
3 & Voice & 3 & 7 & 2 \\
\hline
\end{tabular}




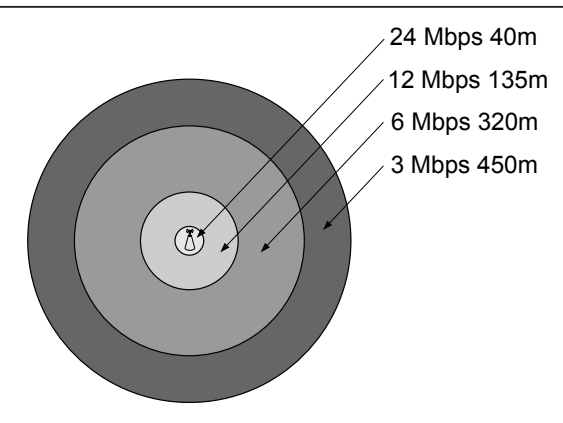

Figure 2 Transmission ranges for different data rates.

transmission rates according to their distance to the RSU. Each vehicle can transmit frames from data classes $\mathrm{AC} k, k=0 . .3$, in either $\mathrm{CCH}$ or $\mathrm{SCH}$. We assume that there are $d_{c}$ data combinations on $\mathrm{CCH}$ and $d_{s}$ data combinations on SCH. Data combination $d_{x}$ on channel $x \in(c, s)$ is characterized with an array of $[c(x, d, 0) c(x$, $d, 1) c(x, d, 2) c(x, d, 3)]$. The index value $c(x, d, k)=1$ denotes the presence of data class $k$, while the value of $c$ $(x, d, k)=0$ denotes its absence. Packets from data class $k$ in channel $x$ arrive to the vehicle according to a Poisson process with rate $\lambda_{x, k}$. Time unit in our model is one backoff slot. For data class $k$ within channel $x$ at each region $\left(R g_{r}\right)$, we assume variable frame size of $l d_{x, k}$, $r$ slots which includes payload, MAC header, and physical header. The PGF for frame size within the transmission range $L$ of the RSU is

$$
L d_{x, k}(z)=\sum_{r=1}^{R g_{\max }} \frac{l_{r}}{L} z^{l d_{x, k, r}}
$$

where $l_{r}$ is the length of the region $R g_{r}$. Duration of the SIFS period in slots will be denoted as sifs. We assume that RTS/CTS transmission scheme is used. Duration of RTS, CTS, and ACK frames expressed in slots will be denoted as rts, cts, and ack, respectively. We model the channel errors through Bit Error Rate (ber). In each region, the probability that a frame will not be corrupted by noise is denoted as $\delta_{k, r}=(1-b e r)^{r t s_{b}+c t s_{b}+l d_{b, x, k, r}+a c k_{b}}$ where subscript $b$ denotes values expressed in bits. Also, we consider
OBU devices equipped with a single networking interface only. Each interface switches between $\mathrm{CCH}$ and $\mathrm{SCH}$ during the sync interval, as shown in Figure 4. Duration of synchronization interval is $S I=100 \mathrm{~ms}$ and duration of guard intervals is $\mathrm{grd}=5 \mathrm{~ms}$ each, as specified in the standard [13]. Duration of $\mathrm{CCH}$ is (SI - 2grd)SO. We assume that duty cycle of $\mathrm{CCH}$ vs. $\mathrm{SCH}$ is $S O=0.5$. For this value, backoff process should be completed within $(S I / 2) \mathrm{ms}$ in each channel in order to avoid handover for another RSU [3]. The handover probability in each channel interval for any packets from data class $k$ is

$$
h d_{x, k}=\frac{(S I / 2) v}{L} S O\left(1-e^{\left.(S I / 2) \lambda_{x, k}\right)} \leq 0.0004\right.
$$

where $v$ is the mean speed of the vehicle. The handover probability is neglected because of its small value and we assume that the backoff process will be completed in the vicinity of a single RSU.

We assume that vehicle's buffer has infinite length and use an $M / G / 1$ queuing model. The PGF for successful packet transmission time is

$$
S t_{x, k}(z)=z^{r t s+c t s+3 s i f s+a c k} \sum_{r=1}^{R g_{\max }} \frac{l_{r}}{L} z^{l d_{x, k, r}}
$$

In case of collision of RTS packets, activity on the medium has the PGF of $C t(z)=\mathrm{z}^{r t s+c t s+s i f s}$.

\subsection{Distribution of vehicles}

The number of vehicles in each lane of road segment follows a Poisson distribution. Let $F$ be the vehicle flow which corresponds to the number of vehicles that pass a fixed roadside point per unit time. Also, let $\lambda_{d}$ be vehicle density, i.e., the number of vehicles per unit distance in one direction along the road segment. From the traffic flow theory [4], mean speed, flow, and density are related through is

$$
F=v \lambda_{d}
$$

Figure 5 shows the relationships among these parameters. The solid portion of the curves represents stable

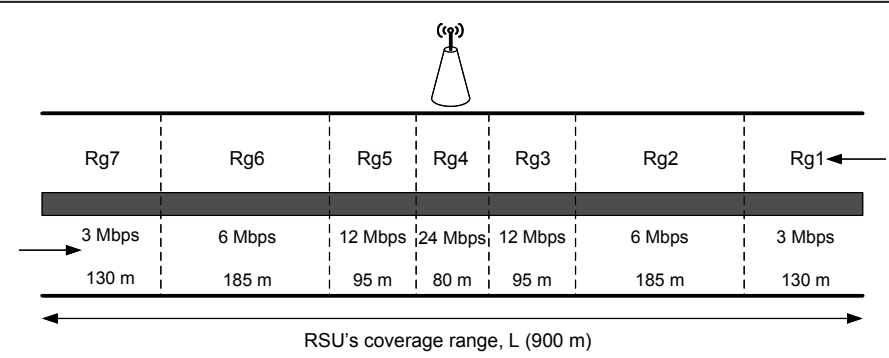

Figure 3 Road segment. 


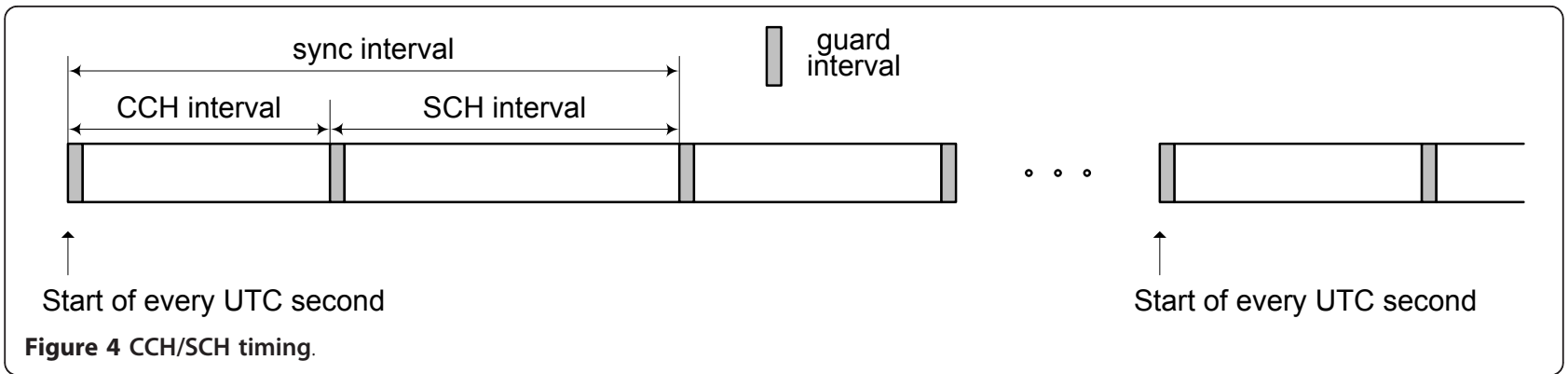

or free-flow state. This state holds until the vehicle density reaches a critical value. The peak of the curves is the maximum rate of flow or the capacity of road segment. Beyond the critical density, some breakdown locations appear on the road segment, which lead to the formation of some queues of vehicles. This state is called unstable or forced flow and is shown by the dashed portion of the curves. If the density increases, the traffic reaches the jam state, at which vehicles have to completely stop [7].

Moreover, Greenshield's model [6] captures the dependency between speed and density by assuming a liner relationship as follows:

$$
v=v_{f}\left(1-\lambda_{d} / \lambda_{d, \mathrm{jam}}\right)
$$

where $v_{f}$ is the free-flow speed corresponding to the maximum desired speed (usually taken as the road's speed limit). $\lambda_{d \text {,jam }}$ is the maximum allowable traffic density (jam density). Then, the maximum number of vehicles, $v h_{\max }$, in each lane of the road segment within the transmission range $L$ of the RSU can be calculated as

$$
v h_{\max }=L \lambda_{d, j a m}
$$

The probability that there are $n$ vehicles in each lane of the road segment is given by

$$
P(n)=\frac{e^{-L \lambda_{d} \frac{\left(L \lambda_{d}\right)^{n}}{n !}}}{\sum_{i=0}^{v h_{\max }} e^{-L \lambda_{d} \frac{\left(L \lambda_{d}\right)^{i}}{i !}}}
$$

\subsection{Model of IEEE $802.11 p$ features}

Let us now briefly review AIFSN-based prioritization, as shown in Figure 6. If the medium gets busy during the backoff countdown, the backoff counter will be frozen until the medium becomes idle again for the uninterrupted duration of aifs $s_{x, k}=$ sifs $+\operatorname{AIFSN}_{x, k} \cdot \sigma$ where $\sigma$ is the duration of the slot time and $x \in(c, s)$ denotes the channel type. We model this action through $k$ freezing counters [14]. They need to be restarted from the beginning if the medium becomes busy at any time during the countdown. Since access is synchronized with the end of previous transmission, no access is possible during $\operatorname{AIFS}_{x, 3}$. Figure 7 presents the Markov chain for freezing countdown, the initial values of freezing counters are set to $B_{x, k}=A I F S N_{x, k}$ $\operatorname{AIFSN}_{x, 3}, k=0 . .3$.

When the backoff count reaches zero, the transmission starts. If a collision occurs, backoff procedure has to be repeated but with increased contention window size. There are $m_{x, k}+1$ backoff phases, starting from the phase 0 , with increasing values of contention window. Frame can be re-transmitted up to $R$ times, but window size will grow only until phase $m_{x, k}$ as indicated in Tables 1 and 2 . The size of contention window for channel $x \in(c, s)$ and $A C=k$ in the $i$ th backoff stage $\left(i=0 . . m_{x, k}\right)$ has the value of

$$
W_{x, k, i}= \begin{cases}2^{i}\left(C W \min _{x, k}+1\right)-1, & 0 \leq i \leq m_{x, k} \\ 2^{m_{k, L}}\left(C W \min _{x, k}+1\right)-1, & R \geq i>m_{x, k}\end{cases}
$$

As shown in Figure 6, duration of periods where data classes $k$ and higher can access channel $x$ are denoted as

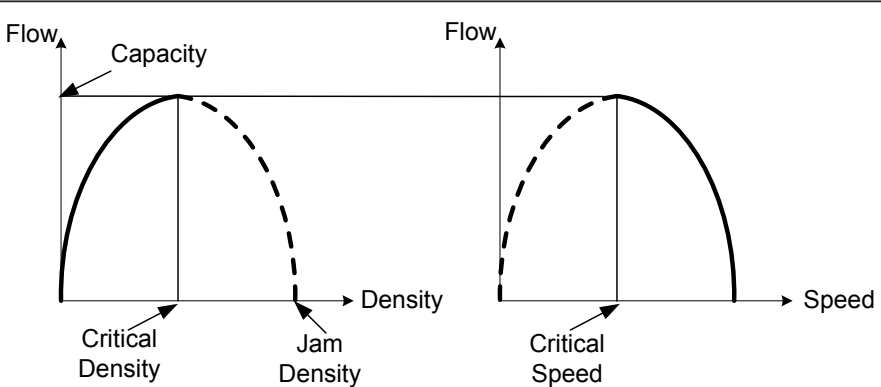

Figure 5 Relationships between flow, speed, and density [4]. 


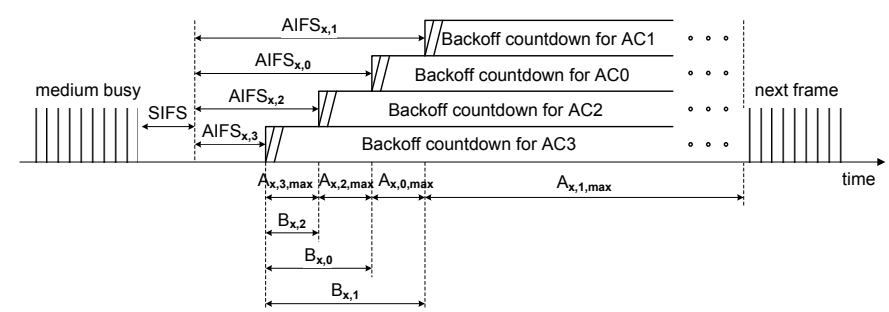

Figure 6 EDCA channel prioritized access for IEEE 802.11p

$A_{x, k}$ and their maximum durations are

$$
A_{x, k, \max }= \begin{cases}\operatorname{AIFSN}_{x, k-1}-\operatorname{AIFSN}_{x, k}, & k=1 . .3 \\ W_{x, 0, \max }, & k=0\end{cases}
$$

Because of internal collisions of frames from different data classes within the vehicle itself, probability that a frame has completed the backoff process (we will denote it as virtual access probability, $\left.\theta_{x, d, k}\right)$ might be different from access probability $\tau_{x, d, k}$ :

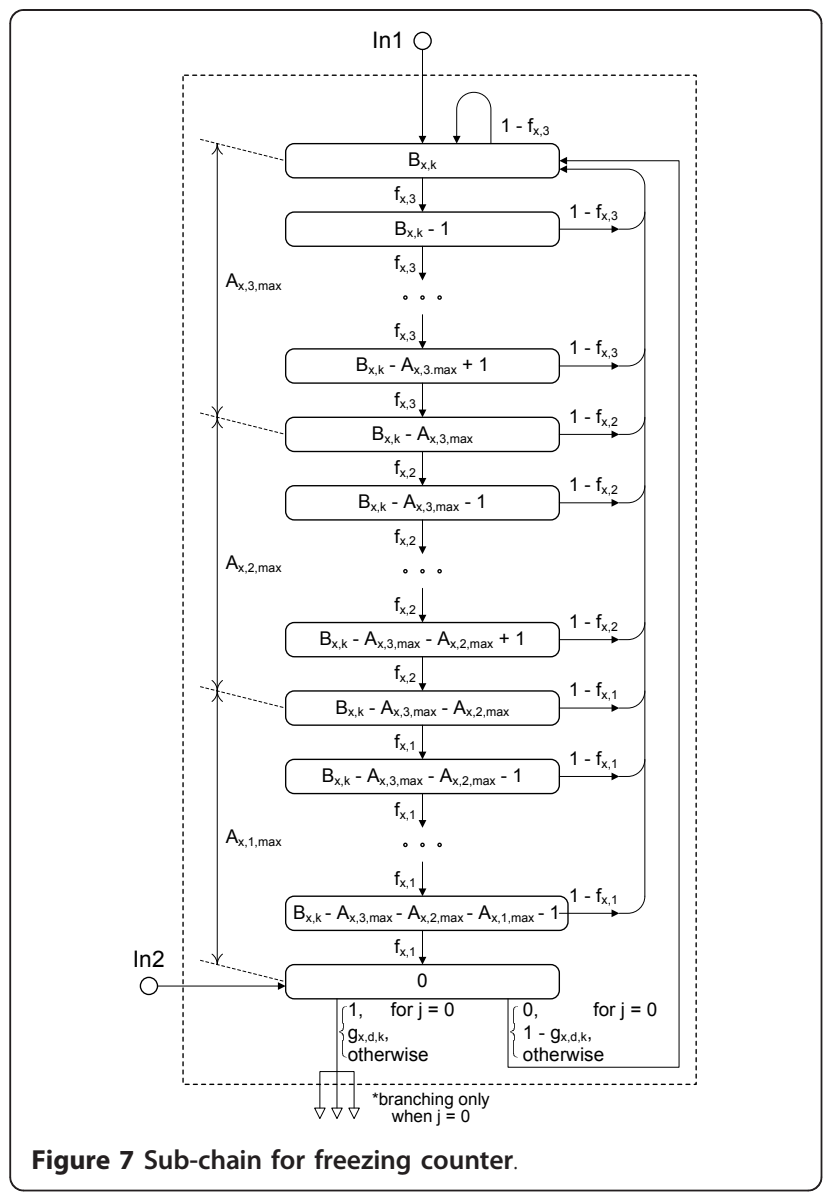

$$
\begin{aligned}
& \tau_{x, d, 3}=\theta_{x, d, 3} \\
& \tau_{x, d, k}=\theta_{x, d, k} \prod_{l=k+1}^{3}\left(1-c(x, d, k) \theta_{x, d, l}\right), \quad k=0 . .2
\end{aligned}
$$

Let the probability that a backoff period is the last in the target channel be $\mathcal{X}_{c}=\frac{1}{c c h}$ and $\mathcal{X}_{s}=\frac{1}{s c h}$ for $\mathrm{CCH}$ and $\mathrm{SCH}$, respectively. Probability that the medium will be idle during a single backoff slot in the target channel interval $A_{x, k}$ is

$$
f_{x, k}=\left(1-\mathcal{X}_{k}\right) \prod_{t=1}^{t_{x}} \prod_{l=k}^{3}\left(1-c(x, d, k) \tau_{x, d, l}\right)^{n_{x, d, l}}
$$

where $n_{x, d, k}$ is the total number of vehicles from channel $x \in c, s$ with data combination $d$, transmitting frames from data class $k$. However, because of virtual collisions, what the stream $c(x, d, k)=1$ actually 'sees' is that no data class within the vehicle has finished the backoff process and that there is no access by any other vehicle. This value might be different from $f_{x, k}$, since it observes absolute access of data classes outside the vehicle and virtual access of classes inside the vehicle as

$$
\zeta_{x, d, k}=f_{x, k} \frac{\prod_{l=k+1}^{3}\left(1-c(x, d, k) \theta_{x, d, l}\right)}{\prod_{l=k+1}^{3}\left(1-c(x, d, k) \tau_{x, d, l}\right)}
$$

Backoff counter for stream $c(x, d, k)=1, x \in(c, s)$, can be decremented during time periods $A_{x, l}, l=0 . . k$, but unfortunately, with probabilities that depend on the index of the period. The overall probability of backoff counter decrement for $A C_{i}$ is

$$
\begin{aligned}
g_{x, d, 0}= & \frac{\zeta_{x, d, 0}}{1-\theta_{x, d, 0}} \\
g_{x, d, i}= & \frac{\left(1-\zeta_{x, d, i}^{A_{x, i} \max }\right) \zeta_{x, d, i}}{1-\theta_{x, d, i}}+\frac{\zeta_{x, d, i}^{A_{x, i \max }}\left(\zeta_{x, d, i}-1\right)}{1-\theta_{x, d, i}}, i=1 . .3
\end{aligned}
$$

The probability that a transmission for ACk in channel $x$ does not experience a virtual or real collision is $\gamma_{n_{x, d, k}}=g_{x, d, k}$. The overall collision probability per lane for data combination $d$ with data class $k$ in channel $x$ 
can be calculated as

$$
E\left[\operatorname{col}_{x, d, k}\right]=1-\sum_{n=0}^{v h_{\max }} P(n) \gamma_{n_{x, d, k}}
$$

\subsection{PGF for the frame service time}

The basic building block of the packet service time PGF is the transfer PGF for the pass through the freezing counter block which has loopback when the value of backoff counter is non-zero, and skips loopback when the backoff counter is zero. Note that transfer PGFs through the block are different if the block has to be traversed vertically from higher backoff state or laterally from the same backoff state but from higher value of backoff counter.

Let us first consider vertical traversal through the freezing counter, in which case we need to derive the probabilities $P f a_{1}$ that freezing counters of vehicles with data classes $k<l$ will be restarted because of successful transmission on the medium in the period $A_{x, l}, l=1,2,3$.

$$
P f a_{x, l}=\sum_{d=1}^{d_{x}} \sum_{i=1}^{3} \frac{n_{x, d, i} \tau_{x, d, i} \prod_{i=l}^{3}\left(1-\tau_{x, d, i}\right)^{n_{x, d, i}}}{\left(1-\tau_{x, d, i}\right)}
$$

The probability that freezing counter will be restarted because of a collision is $P f c_{x, l}=1-f_{x, l}-P f a_{x, l}$.

We also need to derive duration of successful access to the medium during period $A_{x, l}, l=0 . .3$ as

$$
S w_{x, l}(z)=\frac{\sum_{d=1}^{d_{x}} \sum_{i=1}^{3} n_{x, d, i} \tau_{x, d, i} S t_{x, k}(z)}{\sum_{d=1}^{d_{x}} \sum_{i=1}^{3} n_{x, d, i} \tau_{x, d, i}}
$$

Transfer PGFs $B f n l_{x, k}(z)$ for the zero value of backoff counter can be derived by noting that PGF is recursive because of the presence of a loop and has the form $B f n l_{x, k}(z)=B f n l n_{x, k}(z) / B f n l d_{x, k}(z)$ where

$$
\begin{aligned}
B f n l n_{x, k}(z) & =z^{a i f s_{x, 3}} \prod_{i=k+1}^{3}\left(f_{x, i} z\right)^{A_{x, i, \max }} \\
B f n l d_{x, k}(z) & =1-\sum_{i=k+1}^{3}\left(P f a_{x, i} S w_{x, i}(z)+P f c_{x, i} C t(z)\right) \\
& \sum_{n=0}^{A_{x, i, m a x}-1}\left(z f_{x, i}\right)^{n} z \prod_{l=i+1}^{3}\left(z f_{x, l}\right)^{A_{x, l, m a x}}
\end{aligned}
$$

except for the highest traffic class where $B f n l_{x, 3}(z)=z$. In order to calculate vertical transfer PGFs for nonzero values of backoff counter, we need the probabilities that the backoff count will be suppressed because of successful transmission or collision. Probability that the backoff count for traffic class $k$ will be suppressed in the period $A_{x, l} l l=0 . . k$, because of successful transmission is

$$
\begin{aligned}
P b s_{x, d, k, l} & =\sum_{i=l}^{3} \frac{n_{x, d, i} \tau_{x, d, i} \prod_{i=1}^{3}\left(1-\tau_{x, d, i}\right)^{n_{x, d, i}}}{\left(1-\tau_{x, d, k}\right)\left(1-\tau_{x, d, i}\right)} \\
& -\frac{\tau_{x, d, k}}{\left(1-\tau_{x, d, k}\right)^{2}} \prod_{i=l}^{3}\left(1-\tau_{x, d, i}\right)^{n_{x, d, i}}
\end{aligned}
$$

The corresponding probabilities that the backoff count will be suppressed because of the collision on the medium are $P b c_{x, d, k, l}=1-\frac{f_{x, l}}{1-\tau_{x, d, l}}-P b s_{x, d, k, l}$.

Then, the vertical transfer PGF for non-zero value of backoff counter gets the form $B f l_{x, d, k}(z)=\alpha / \beta$ where $\alpha=c(x, d, k) \frac{f_{x, k}}{1-\tau_{x, t, k}} z B f n l_{x, k}(z)$ and $\beta=1-z B f n l_{x, k}(z)$ $\left(P b s_{x, d, k} S w_{x, k}(z)+P b c_{x, d, k} C t(z)\right)$.

Lateral transfer PGFs which connect the backoff freezing blocks, $B f s_{x, d, k}(z)$, can be calculated as defined in [3].

The PGF for the duration of the backoff phase $i$ for data class $k$ on channel $x$ is

$$
B_{x, d, k, i}(z)=\frac{B f n l_{x, k}(z)}{W_{x, k, i}}+\frac{B f l_{x, d, k}(z)}{W_{x, k, i}} \sum_{l=1}^{W_{x, k, i}-1} B f_{s_{x, d, k}}(z)^{l}
$$

The probability that the OBU's buffer is empty at arbitrary time is $\pi_{x, d, k, 0}=1-\rho_{x, d, k}$, where $\rho_{x, d, k}$ denotes offered load from data combination $d$ with class $k$ in channel $x$. The entire Markov chain for $A C_{k}$ is shown in Figure 8. Markov chain has the same form for both channels since activity of the other channel is equivalent to channel busy state on the current channel. By considering Figure 8, the PGF for the backoff time for data combination $d$ with data class $A C_{k}$ in channel $x$ becomes

$$
\begin{aligned}
B o f_{x, d, k}(z) & =\sum_{r=1}^{R g_{\max }} \frac{l_{r}}{L}\left(\sum_{i=1}^{m_{x, k}+1}\left(\prod_{j=0}^{i-1} B_{x, d, k, j}(z)\right)\right. \\
& \cdot\left(1-\delta_{k, r} \gamma_{x, d, k}\right)^{i-1} C t(z)^{i-1} \delta_{k, r} \gamma_{x, d, k} \\
& +\sum_{i=m_{x, k}+1}^{R}\left(\prod_{j=0}^{m_{x, k}} B_{x, d, k, j}(z)\right) B_{x, d, k, m_{x, k}}(z)^{i-m_{x, k}} \\
& \cdot\left(1-\delta_{k, r} \gamma_{x, d, k}\right)^{i} C t(z)^{i} \delta_{k, r} \gamma_{x, d, k} \\
& \left.+\left(\prod_{j=0}^{m_{x, k}} B_{x, d, k, j}(z)\right) B_{x, d, k, m_{x, k}}(z)^{R-m_{x, k}}\right) \\
& \left.\cdot\left(1-\delta_{k, r} \gamma_{x, d, k}\right)^{R+1} \operatorname{Ct}(z)^{R+1}\right)
\end{aligned}
$$




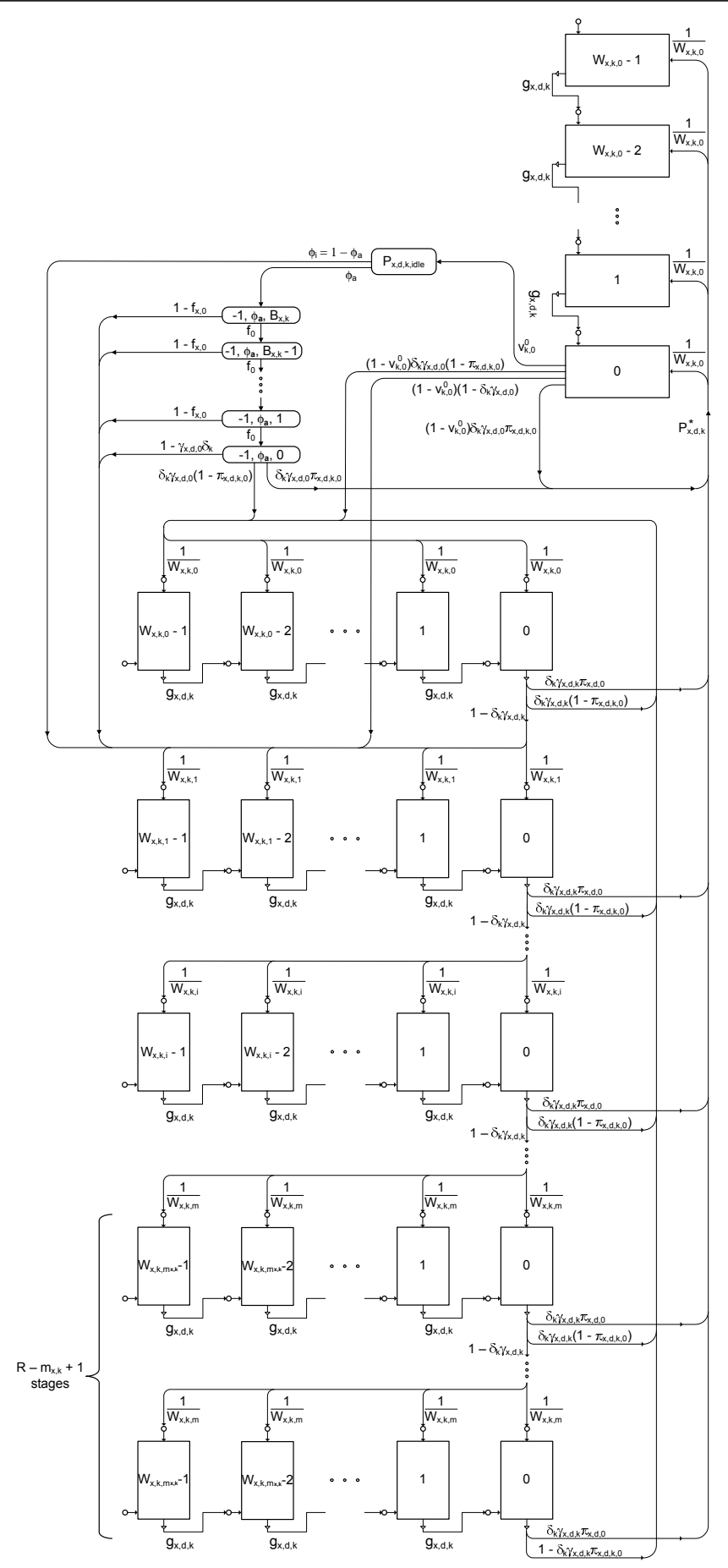

Figure 8 Markov chain for $A C_{k}$ in channel $x$

When the buffer is found empty upon a successful packet transmission, the vehicle can proactively undertake zeroth backoff, go to the idle state, and then attempt to transmit the next arriving packet upon waiting only for $A I F S_{x, k}$. However, if this attempt is not successful, the entire backoff process (beginning from phase 1) has to be performed; the PGF for the duration of this backoff process, $B z o f_{x, d, k}(s)$, is similar 
to the one in (18) but with a difference in the first line:

$$
\begin{aligned}
B z o f_{x, d, k}(z) & =\sum_{r=1}^{R g_{\max }} \frac{l_{r}}{L}\left(\gamma_{x, d, k \delta_{k, r}}+\sum_{i=2}^{m_{x, k}+1}\left(\prod_{j=0}^{i-1} B_{x, d, k, j}(z)\right)\right. \\
& \cdot\left(1-\delta_{k, r} \gamma_{x, d, k}\right)^{i-1} C t(z)^{i-1} \delta_{k, r} \gamma_{x, d, k} \\
& +\sum_{i=m_{x, k}+1}^{R}\left(\prod_{j=0}^{m_{x, k}} B_{x, d, k, j}(z)\right) B_{x, d, k, m_{x, k}}(z)^{i-m_{x, k}} \\
& \cdot\left(1-\delta_{k, r} \gamma_{x, d, k}\right)^{i} C t(z)^{i} \delta_{k, r} \gamma_{x, d, k} \\
& \left.+\left(\prod_{j=0}^{m_{x, k}} B_{x, d, k, j}(z)\right) B_{x, d, k, m_{x, k}}(z)^{R-m_{x, k}}\right) \\
& \left.\cdot\left(1-\delta_{k, r} \gamma_{x, d, k}\right)^{R+1} \operatorname{Ct}(z)^{R+1}\right)
\end{aligned}
$$

At this point, these expressions can be used to calculate extended backoff times Bofe $e_{c, d, k}(z)$ and $B z o f e_{c, d, k}(z)$, as defined in [3].

Markov chain presents a random process with stationary distribution $y_{x, d, k, i, j, b}$, where $x \in(c, s), d=1 . . d_{x}$ denotes vehicle type; $k=0 . .3$ denotes the data class, $i=$ $0 . . m_{k}$ denotes the index of the backoff phase, $j=0 . . W_{x}$, $k, i-1$ denotes the value of backoff counter, and $b=0$.. $B_{k}$ denotes the value of the freezing counter.

In order to model behavior of vehicle after the successful transmission we need to note that standard requires vehicle to perform backoff with $W_{x, k, 0}$ immediately after successful transmission even if the vehicle's buffer is empty. If vehicle's buffer is still empty after this backoff count, vehicle enters idle state represented by $P_{\text {idle }}$ in Figure 8 .

The total idle state probability is calculated by finding the distance between two accesses by the same vehicle. In order to obtain this result we use Laplace-Stieltjes Transform (LST) of backoff time PGF which is obtained as $B o f e_{x, d, k}^{*}(s)=B o f e_{x, d, k}\left(e^{-s}\right)$. If we introduce probability of no frame arrivals during zeroth backoff on channel $x$ as $v_{x, d, k, 0}^{0}$ and LST of backoff process without the zeroth backoff phase as $B z o f e_{x_{1}, d, k}^{*}(s)$ the distance between two transmissions becomes

$$
\begin{aligned}
D_{x, d, k}^{*}(s) & =\sum_{r=1}^{R g_{\max }} \frac{l_{r}}{L}\left(S t _ { x , k } ( e ^ { - s } ) \left\{\pi_{x, d, k, 0} B_{x, d, k, 0}\left(e^{-s}\right)\right.\right. \\
& v_{x, d, k, 0}^{0} R_{x, k}^{*}(s)\left[\phi _ { i } I _ { x } ^ { * } ( s ) \left(\prod_{l=k+1}^{3} f_{x, l}^{A_{x, l m a x}} \delta_{k, r}\right.\right. \\
& \left.+\left(1-\prod_{l=k+1}^{3} f_{x, l}^{A_{x, l, m a x}} \delta_{k, r}\right) B o f e_{x, d, k}^{*}(s)\right)+\phi_{a}\left(f_{x, 0}^{a i f s_{x, k}} \delta_{k, r}(20)\right. \\
& \left.\left.+\left(1-f_{x, 0}^{a i f s_{x, k}} \delta_{k, r}\right) B o f e_{x, d, k}^{*}(s)\right)\right] e^{-s a i f s_{x, k}} \\
& +\pi_{x, d, k, 0} B_{x, d, k, 0}\left(e^{-s}\right)\left(1-v_{x, d, k, 0}^{0}\right)\left(\gamma_{x, d, k} \delta_{k, r}\right. \\
& \left.+\left(1-\gamma_{x, d, k} \delta_{k, r}\right) B z o f e_{x, d, k}^{*}(s)\right) \\
& \left.\left.+\left(1-\pi_{x, d, k, 0}\right) \operatorname{Bof} e_{x_{,}, d, k}^{*}(s)\right\}\right)
\end{aligned}
$$

where $R_{x, k}^{*}(s)=\frac{\lambda_{x, k}}{\lambda_{x, k}+s}$ is the LST for exponential distribution of the residual frame inter-arrival time and $I_{x}^{*}(s)$ denotes LST of time needed to synchronize with the beginning of $\mathrm{CCH}$ or $\mathrm{SCH}$ period [3]. $\varphi_{a}$ is the probability that the target channel is active when the vehicle exits idle state and $\varphi_{i}$ is the probability that the opposite channel (or guard time) is active. Then, the LST for the total active time between two successive access point, i.e., frame service time is

$$
\begin{aligned}
& T t_{x, d, k}^{*}(s)=\sum_{r=1}^{R g_{\max }} \frac{l_{r}}{L}\left(S t _ { x , k } ( e ^ { - s } ) \left\{\pi_{x, d, k, 0} B_{x, d, k, 0}\left(e^{-s}\right)\right.\right. \\
& v_{x, d, k, 0}^{0}\left[\phi _ { i } I _ { x } ^ { * } ( s ) \left(\prod_{l=k+1}^{3} f_{x, l}^{A_{x l, m a x}} \delta_{k, r}\right.\right. \\
& \left.+\left(1-\prod_{l=k+1}^{3} f_{x, l}^{A_{x, \max }} \delta_{k, r}\right) B o f e_{x, d, k}^{*}(s)\right)+\phi_{a}\left(f_{x, 0}^{a i f f_{s, k}} \delta_{k, r}\right. \\
& \left.\left.+\left(1-f_{x, 0}^{a i f s_{x, k}} \delta_{k, r}\right) B o f e_{x, d, k}^{*}(S)\right)\right] e^{-s a i f s_{x, k}} \\
& +\pi_{x, d, k, 0} B_{x, d, k, 0}\left(e^{-s}\right)\left(1-v_{x, d, k, 0}^{0}\right)\left(\gamma_{x, d, d} \delta_{k, r}\right. \\
& \left.+\left(1-\gamma_{x, d, k} \delta_{k, r}\right) B z o f e_{x, d, k}^{*}(s)\right) \\
& \left.\left.+\left(1-\pi_{x, d, k, 0}\right) B o f e_{x, d, k}^{*}(s)\right\}\right)
\end{aligned}
$$

and its first two moments are $\overline{T t_{x, d, k}}=\left.\frac{d T t_{x, d, k}^{*}(s)}{d s}\right|_{s=0}$ and $\overline{T t_{x, d, k}^{(2)}}=\left.\frac{d^{2} T t_{x, d, k}^{*}(s)}{d s^{2}}\right|_{s=0}$. Offered load on the channel now becomes $\rho_{x, d, k}=\lambda_{x, k} \overline{T t_{x, d}, k}$.

The probability that the vehicle is idle can be calculated as $P_{x, d, k, i d l e}=1-\frac{\overline{T t_{x, d, k}}}{\overline{D_{x, d, k}}}$. Now we can form the equation for normalization condition as defined in [3].

\subsection{Throughput and waiting time for $A C_{k}$ in channel $x$}

Normalized throughput per vehicle for data combination $d$ with data class $k$ in channel $x$ can be obtained

as

$$
T h r_{n_{x, d, k}}=\frac{\overline{l d_{x, k}}}{\overline{D_{x, d, k}}}
$$

The overall normalized throughput per lane can be calculated as

$$
E\left[T h r_{x, d, k}\right]=\sum_{n=0}^{v h_{\max }} P(n) T h r_{n_{x, d, k}}
$$

The PGF for the number of frame arrivals during frame service time is $A_{x, d, k}(z)=T t_{x, d, k}^{*}\left(\lambda_{x, k}(1-z)\right)$, and the PGF for the number of frames left after the departing frame in the vehicle buffer is [15] 


$$
\prod_{x, d, k}(z)=\frac{\pi_{x, d, k, 0}(1-z) A_{x, d, k}(z)}{A_{x, d, k}(z)-z}
$$

In order to calculate the response time, we observe that number of frames left in the buffer after the departing frame is equal to the number of frames which arrived while the frame was buffered and serviced. If we denote the LST of the response time for $A C_{k}$ in channel $x$ as $W_{x, d, k}^{*}(s)$, last statement can be written as $\prod_{x, d, k}(z)=W_{x, d, k}^{*}\left(\lambda_{x, k}-z \lambda_{x, k}\right) T t_{x, d, k}^{*}\left(\lambda_{x, k}-z \lambda_{x, k}\right)$. Because of PASTA property of M/G/1 systems, $\Pi_{x, d, k}(z)$ also presents probability distribution of buffer occupancy at arbitrary time. After substituting $s=\lambda_{x, k}-z \lambda_{x, k}$, we obtain the LST of the waiting time as $W_{x, d, k}^{*}(s)=\frac{s\left(1-\rho_{x, d, k}\right)}{s-\lambda_{x, k}+\lambda_{x, k} T t_{x, d, k}^{*}(s)}$, and its average value as $\overline{W_{n_{x, d, k}}}=\frac{\lambda_{x, k} \overline{T t_{k}^{(2)}}}{2\left(1-\rho_{x, d, k}\right)}$. Similar to (23), the overall waiting time per lane can be calculated as

$$
E\left[\overline{W_{x, d}, k}\right]=\sum_{n=0}^{v h_{\max }} P(n) \overline{W_{n_{x, d, k}}}
$$

\section{Performance evaluation}

We have evaluated network performance for multiple data classes in a network with a single RSU deployed on a bidirectional road segment with transmission ranges of $L=900,500$, and $350 \mathrm{~m}$, respectively. In order to investigate the network transition between non-saturation and saturation regimes, we have conducted a number of experiments with variable vehicle densities in a single contention domain of IEEE 802.11p. According to the distance of the vehicles from the RSU, data rate varies between 3 and 24 Mbps. OFDMA is chosen as the physical layer modulation mechanism with $40 \mu \mathrm{s}$ as the duration of preamble and header transmission. Slot time is set to $16 \mu \mathrm{s}$ and duration of sifs is equal to 2 slots. Bit error rate is set to ber $=2 * 10^{-5}$. Frame size is set to 500 bytes. RTS/CTS scheme is used for the medium access. Durations of synchronization interval and guard intervals are set to 100 and $5 \mathrm{~ms}$, respectively. Duty cycle of $\mathrm{CCH}$ vs. $\mathrm{SCH}$ is set to 0.5 . We have assumed that there are three data combinations in $\mathrm{CCH}$ and $\mathrm{SCH}$ as shown in Table 3. The maximum allowable

Table 3 Populations of vehicles and their data traffic running on the $\mathrm{CCH}$ and $\mathrm{SCH}$

\begin{tabular}{ccc}
\hline Data combination & Data classes & Number of vehicles \\
\hline 1 & $A C 1$ and $A C 3$ & $20 \%$ of vehicles \\
2 & $A C 0$ and $A C 2$ & $20 \%$ of vehicles \\
3 & $A C 0$ & $60 \%$ of vehicles \\
\hline
\end{tabular}

traffic density, $\lambda_{d \text {,jam }}$, per lane is set to $0.1 \mathrm{veh} / \mathrm{m}$. Data rates for different classes are shown in Table 4.

Using these parameter values, the equations acquired from the analytical model (Markov chain and queuing models) are solved using Maple 13 [16]. In the depicted figures, the values for data classes of best effort $(A C 1)$, backgrounds $(A C 0 \mathrm{~s})$ in data combination 2 and 3 , video $(A C 2)$ and voice $(A C 3)$ are shown with boxes, crosses, dashed lines, circles, and diamonds, respectively.

Figure 9 shows the mean backoff time of data classes to successfully access the medium on $\mathrm{CCH}$ and $\mathrm{SCH}$ where the transmission range of the RSU is equal to 900,500 , and $350 \mathrm{~m}$, respectively. Figure 10 shows the average waiting time in the queue of the data frames of different data classes for $\mathrm{CCH}$ and $\mathrm{SCH}$ where the RSU's transmission range varies. The plots show that as the transmission range increases the network moves from non-saturation regime to saturation regime sooner upon the traffic density growth. Figures 9a, d and 10a, d indicate that in case of having the transmission range $900 \mathrm{~m}$ the saturation regime for the lowest data class priority occurs when the vehicle density is about 0.07 $\mathrm{veh} / \mathrm{m}$ on $\mathrm{CCH}$ and $0.05 \mathrm{veh} / \mathrm{m}$ on $\mathrm{SCH}$. The saturation boundaries are reached for the data classes earlier in the $\mathrm{SCH}$ compared to the $\mathrm{CCH}$ because the data frame arrival rates for $A C 0$ and $A C 1$ are larger in the $\mathrm{SCH}$. However, when the transmission range is equal to 500 and $350 \mathrm{~m}$, the lowest data class priority $(A C 1)$ does not enter into saturation regime before the traffic density is about $0.1 \mathrm{veh} / \mathrm{m}$ per lane. Networking saturation does not occur in the transmission ranges of 500 and $350 \mathrm{~m}$ but spatial saturation occurs when the traffic density per lane becomes equal to $0.1 \mathrm{veh} / \mathrm{m}$. At this traffic density, the traffic flow gradually comes to a stop as a result of the jam density.

Figure 11 shows the collision probability for all data classes where the traffic density increases for the transmission range of 900,500 , and $350 \mathrm{~m}$, respectively. When the vehicle density grows the collision probability increases because the medium contention increases. Comparing the graphs for different transmission ranges shows that a higher transmission range leads to a higher collision probability. As the plots indicate the collision probabilities in the $\mathrm{SCH}$ for all data classes and the RSU's transmission ranges are larger than the corresponding values in the $\mathrm{CCH}$.

\begin{tabular}{lccc}
\multicolumn{4}{l}{ Table $\mathbf{4}$ Data rates per data classes } \\
\hline AC & Data type & On CCH (kbps) & On SCH (kbps) \\
\hline$A C 1$ & Background & 4 & 20 \\
$A C 0$ & Best effort & 4 & 20 \\
$A C 2$ & Video & 12 & 12 \\
$A C 3$ & Voice & 12 & 12 \\
\hline
\end{tabular}




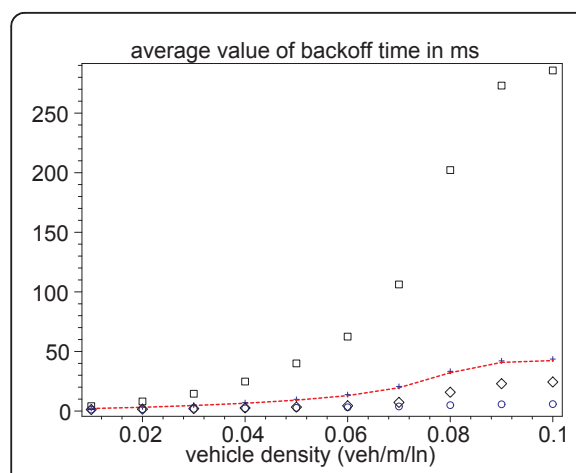

(a) Backoff time for $L=900 \mathrm{~m}(\mathrm{CCH})$.

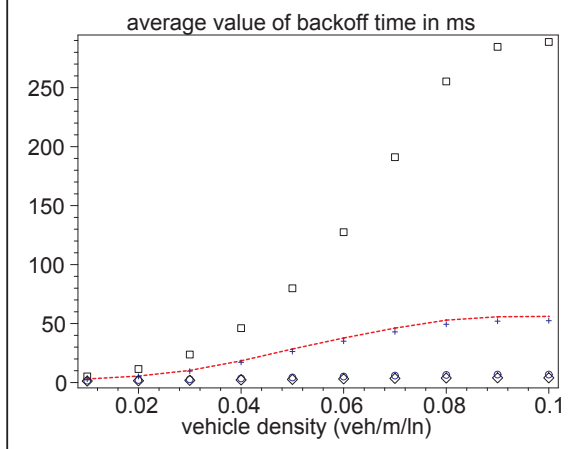

(d) Backoff time for $L=900 \mathrm{~m}$ ( $\mathrm{SCH}$ ).

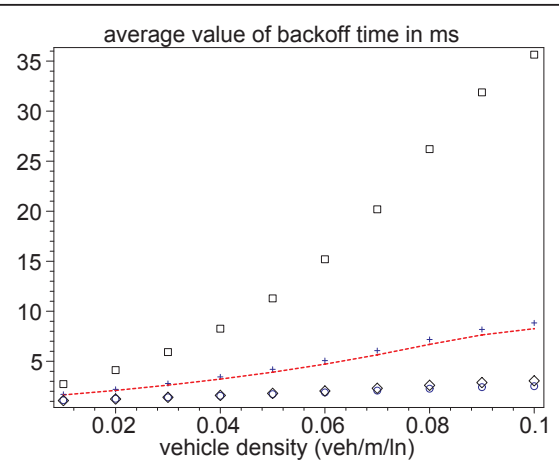

(b) Backoff time for $L=500 \mathrm{~m}$ (CCH).

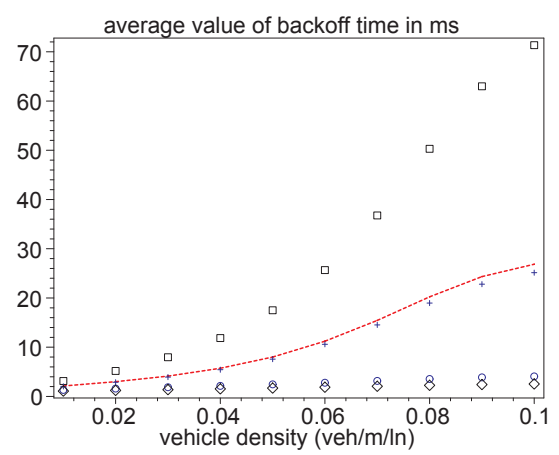

(e) Backoff time for $L=500 \mathrm{~m}(\mathrm{SCH})$.

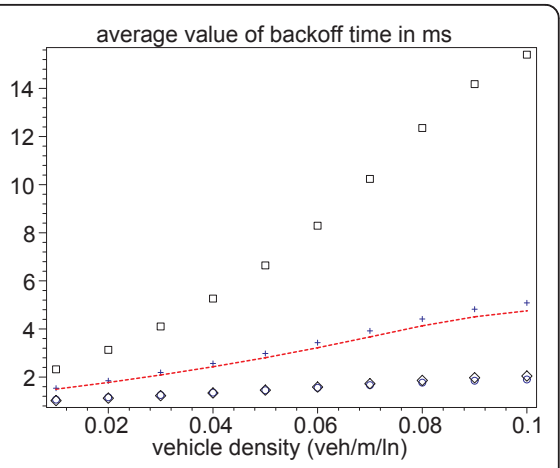

(c) Backoff time for $L=350 \mathrm{~m}(\mathrm{CCH})$.

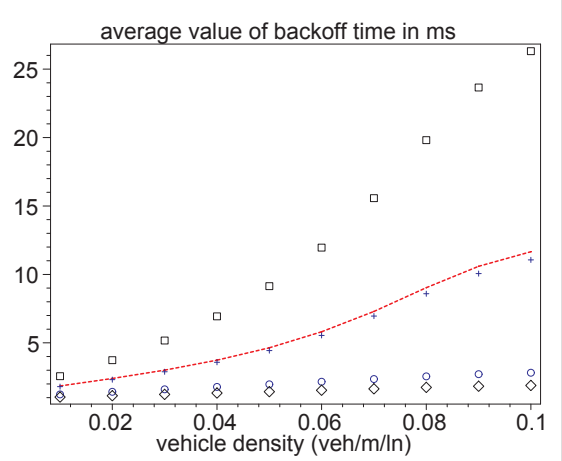

(f) Backoff time for $L=350 \mathrm{~m}$ (SCH).

Figure 9 Average value of backoff time; top row: $C C H$ (a) backoff time for $L=900 \mathrm{~m}(\mathrm{CCH})$, (b) backoff time for $L=500 \mathrm{~m}$ (CCH), (c) backoff time for $L=350 \mathrm{~m}(\mathrm{CCH})$; bottom row: SCH (d) backoff time for $L=900 \mathrm{~m}(\mathrm{SCH})$, (e) backoff time for $L=500 \mathrm{~m}$ (SCH), (f) backoff time for $L=$ $350 \mathrm{~m}(\mathrm{SCH})$.

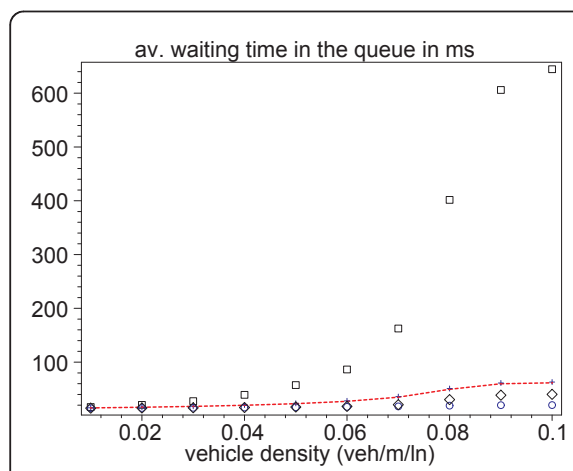

(a) Waiting time for $L=900 \mathrm{~m}(\mathrm{CCH})$.

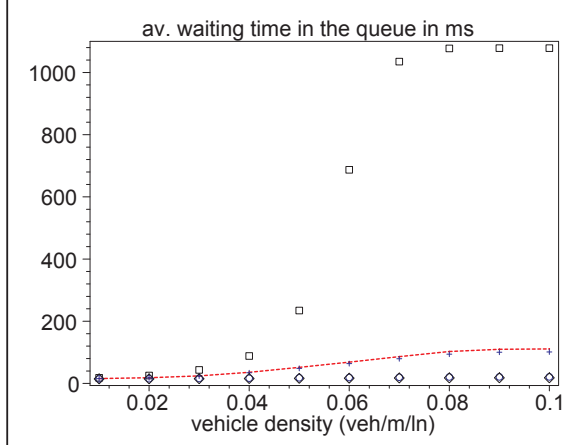

(d) Waiting time for $L=900 \mathrm{~m}(\mathrm{SCH})$.

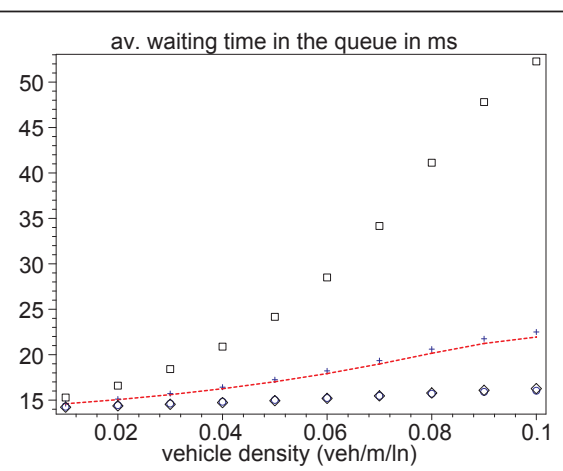

(b) Waiting time for $L=500 \mathrm{~m}(\mathrm{CCH})$.

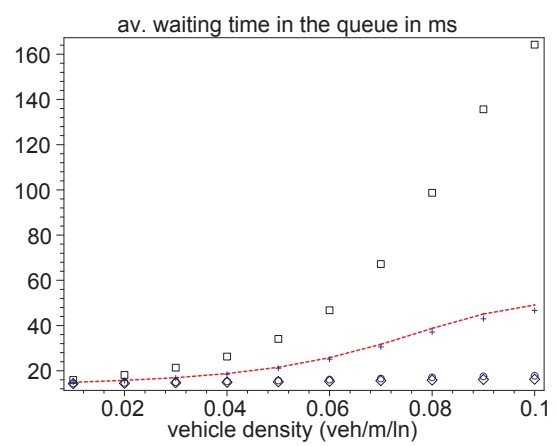

(e) Waiting time for $L=500 \mathrm{~m}(\mathrm{SCH})$.

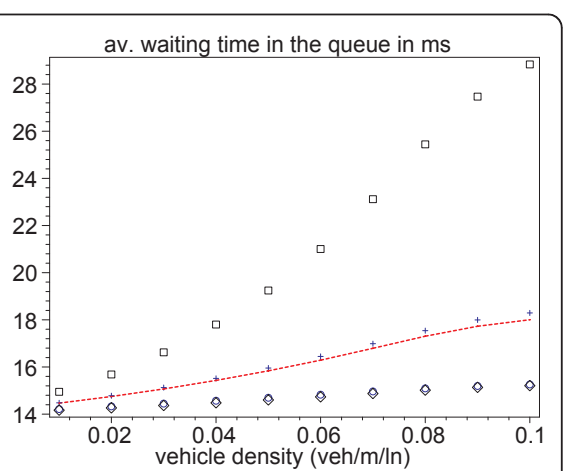

(c) Waiting time for $L=350 \mathrm{~m}(\mathrm{CCH})$.

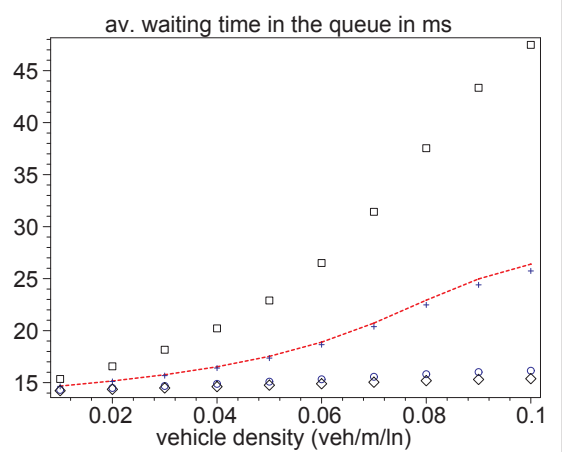

(f) Waiting time for $L=350 \mathrm{~m}(\mathrm{SCH})$.

Figure 10 Average waiting time in the queue; top row: $C C H$ (a) waiting time for $L=900 \mathrm{~m}$ (CCH), (b) waiting time for $L=500 \mathrm{~m}$ (CCH), (c) waiting time for $L=350 \mathrm{~m}(\mathrm{CCH})$; bottom row: $\mathrm{SCH}$ (d) waiting time for $L=900 \mathrm{~m}(\mathrm{SCH})$, (e) waiting time for $L=500 \mathrm{~m}(\mathrm{SCH})$, (f) waiting time for $L=350 \mathrm{~m}(\mathrm{SCH})$. 


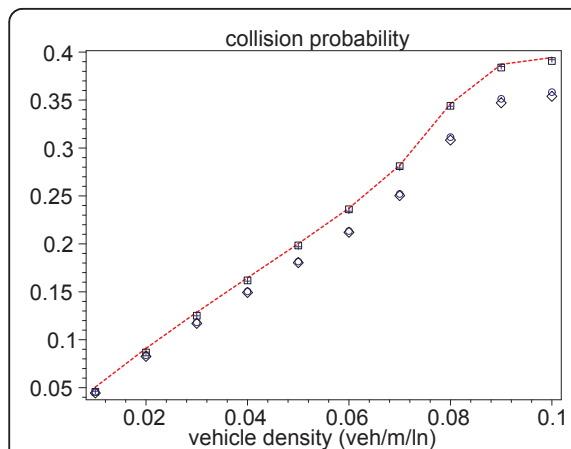

(a) Collision probability for $L=900 \mathrm{~m}(\mathrm{CCH})$.

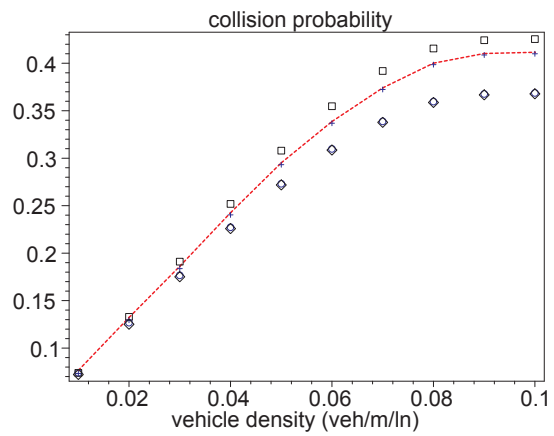

(d) Collision probability for $L=900 \mathrm{~m}(\mathrm{SCH})$.

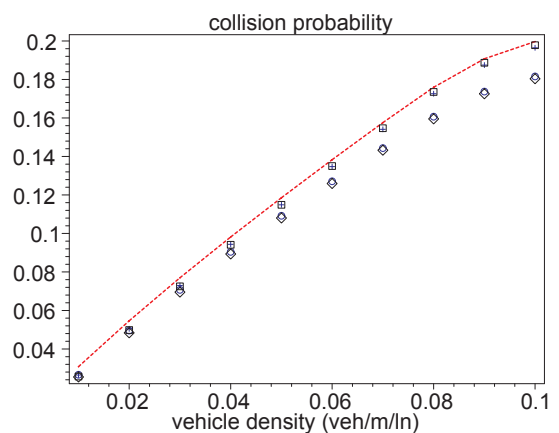

(b) Collision probability for $L=500 \mathrm{~m}(\mathrm{CCH})$.

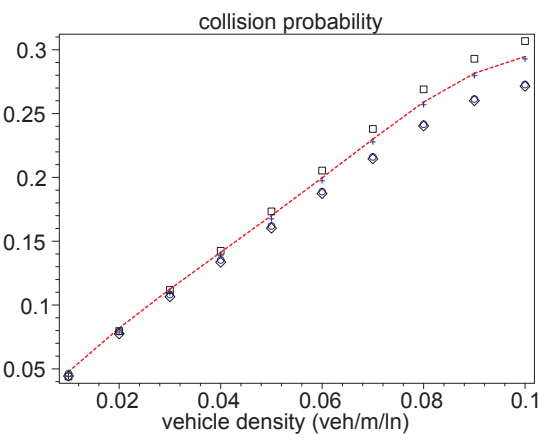

(e) Collision probability for $L=500 \mathrm{~m}(\mathrm{SCH})$.

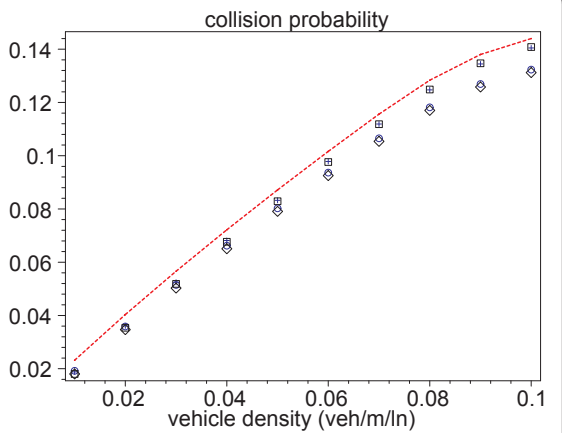

(c) Collision probability for $L=350 \mathrm{~m}(\mathrm{CCH})$.

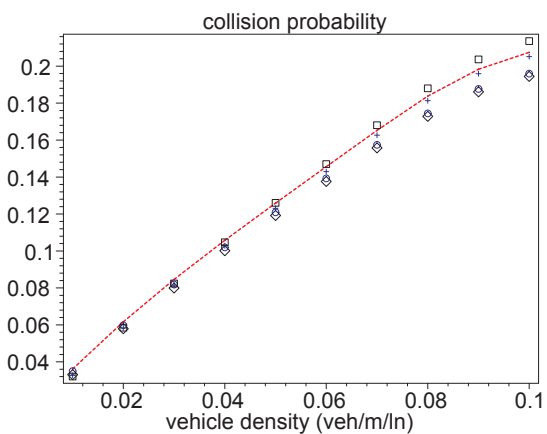

(f) Collision probability for $L=350 \mathrm{~m}(\mathrm{SCH})$.

Figure 11 Collision probability; top row: $C C H$ (a) collision probability for $L=900 \mathrm{~m}(\mathrm{CCH})$, (b) collision probability for $L=500 \mathrm{~m}$ (CCH), (c) collision probability for $L=350 \mathrm{~m}(\mathrm{CCH})$; bottom row: SCH (d) collision probability for $L=900 \mathrm{~m}(\mathrm{SCH})$, (e) collision probability for $L=500 \mathrm{~m}$ $(\mathrm{SCH})$, (f) collision probability for $L=350 \mathrm{~m}(\mathrm{SCH})$.

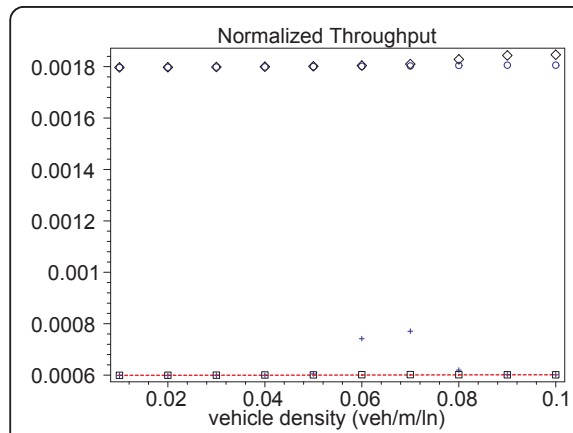

(a) Normalized throughput for $L=900 \mathrm{~m}(\mathrm{CCH})$.

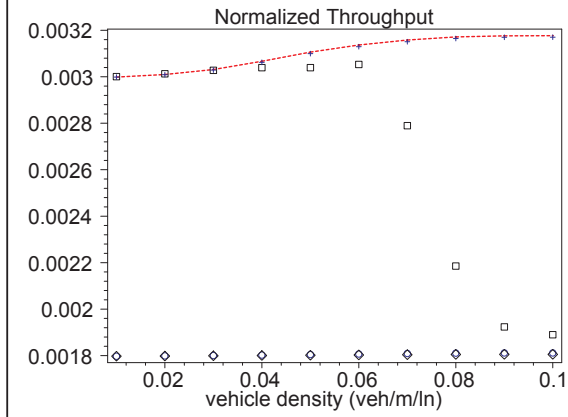

(d) Normalized throughput for $L=900 \mathrm{~m}(\mathrm{SCH})$.

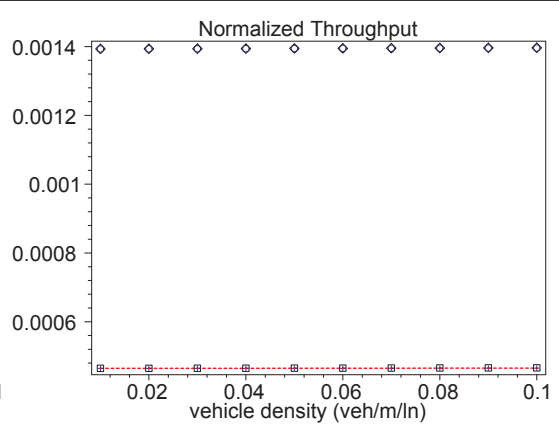

(b) Normalized throughput for $L=500 \mathrm{~m}(\mathrm{CCH})$.

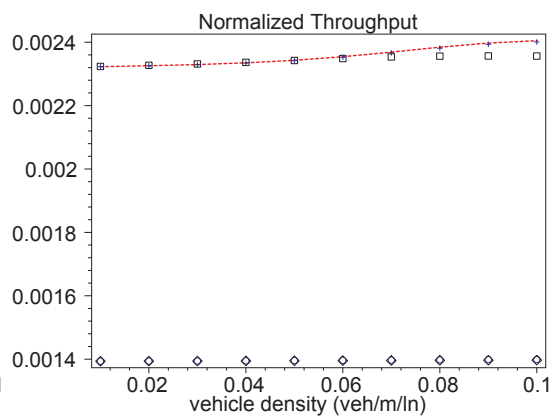

(e) Normalized throughput for $L=500 \mathrm{~m}(\mathrm{SCH})$.

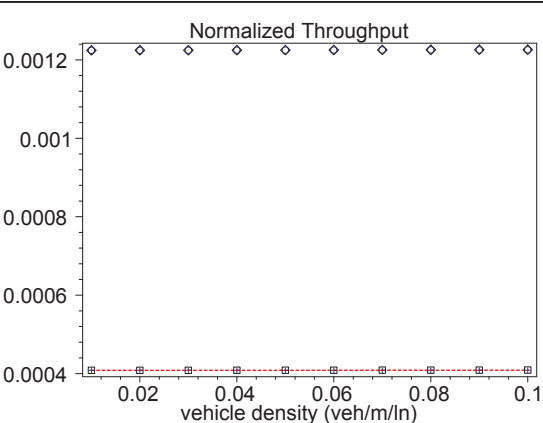

(c) Normalized throughput for $L=350 \mathrm{~m}(\mathrm{CCH})$.

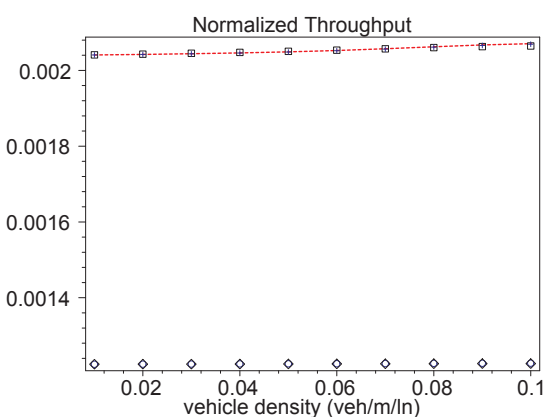

(f) Normalized throughput for $L=350 \mathrm{~m}(\mathrm{SCH})$.

Figure 12 Normalized throughput; top row: $\mathrm{CCH}$ (a) normalized throughput for $L=900 \mathrm{~m}$ (CCH), (b) normalized throughput for $L=500 \mathrm{~m}$ (CCH), (c) normalized throughput for $L=350 \mathrm{~m}(\mathrm{CCH})$; bottom row: $\mathrm{SCH}$ (d) normalized throughput for $L=900 \mathrm{~m}$ (SCH), (e) normalized throughput for $L=500 \mathrm{~m}(\mathrm{SCH})$, (f) normalized throughput for $L=350 \mathrm{~m}(\mathrm{SCH})$. 
In Figure 12, the normalized throughput for all data classes in the $\mathrm{CCH}$ and $\mathrm{SCH}$ are depicted where the transmission range and the vehicle density vary. The results indicate that in the $\mathrm{CCH} A C O$ is the only data class which enters into saturation regime in case of spatial non-saturation. However, in the $\mathrm{SCH}$ only $A C 1$ enters into saturation regime when the traffic density is smaller than $0.1 \mathrm{veh} / \mathrm{m}$ on account of having the lowest priority in the network.

Our results indicate that in each channel lowest priority data classes $(A C 0$ and $A C 1)$ are severely affected by the number of $A C 2$ and $A C 3$ vehicles in the network. The strong prioritization of data classes in the standard is the main reason for the low performance of the low priority data classes.

These performance results would change if duty cycle of $\mathrm{CCH}$ vs. $\mathrm{SCH}$ decreases in order to give more bandwidth to $\mathrm{SCH}$.

\section{Conclusion}

In this article, we studied the spatial and networking transition from non-saturation to saturation regimes for a VANET. We developed an analytical model to investigate the performance of the network with mobile nodes and in non-saturated regime. The model is composed of Markov chain, queuing sub-models, and probabilistic spatial model of vehicles. The model is analytically solved to calculate the performance descriptors of the network, mean backoff time to access the medium, average waiting time in queue, collision probability of a transmission, and normalized throughput, for all data classes. Our study indicates that increasing the transmission range of an RSU leads to earlier networking saturation of the data classes because of increasing the number of contending vehicles in the network. In addition, the results show that highest data classes $(A C 2$ and $A C 3)$ are strongly prioritized over the lowest data classes ( $A C 0$ and $A C 1$ ) inasmuch as the lowest data classes enter into saturation regime much earlier. The vehicle density growth also moves the vehicle traffic from a stable regime to an unstable condition.

In order to optimize the performance of the network in $\mathrm{CCH}$ and $\mathrm{SCH}$ and also balance the entry point to saturation among the channels we need to investigate the duty cycle of a VANET. In our future study, we study how duty cycle of $\mathrm{CCH} / \mathrm{SCH}$ improve the performance of the network. In addition, we will study the optimal deployment of RSUs in a VANET to enhance the network performance. The RSUs' optimal deployment is required because of variable vehicle densities in different geographical areas.

\section{Author details}

${ }^{1}$ Erciyes University, Kayseri, Turkey ${ }^{2}$ Ryerson University, Toronto, ON, Canada

\section{Competing interests}

The authors declare that they have no competing interests.

Received: 29 June 2011 Accepted: 18 November 2011

Published: 18 November 2011

\section{References}

1. Y Zhuang, V Viswanathan, J Pan, L Cai, Upload Capacity Analysis for Drivethru Internet, (Technical report, University of Waterloo, ON, 2010) https:// 129.97.58.88/ojs-2.2/index.php/pptvt/article/view/619/213

2. C Campolo, A Molinaro, Data rate selection in WBSS-based IEEE $802.11 \mathrm{p} /$ WAVE vehicular ad hoc networks. in CSNDSP 2010 412-416 (2010)

3. J Mišsićc, G Badawy, VB Mišsićc, Performance characterization for IEEE 802.11p network with single channel devices. IEEE Trans Veh Technol. 60(4), 1775-1787 (2011)

4. RP Roess, ES Prassas, WR McShane, Traffic Engineering, 3rd edn. (Pearson Prentice Hall, Upper Saddle River, 2004)

5. TV Woensel, $N$ Vandaele, Modeling traffic flows with quequeing models: a review. Asia-Pacific J Oper Res (APJOR). 24(4), 435-461 (2007). doi:10.1142/ S0217595907001383

6. JD Fricker, RK Whitford, Fundamentals of Transportation Engineering: A Multimodal Systems Approach (Prentice Hall, Upper Saddle River, 2004)

7. S Yousefi, E Altman, R El-Azouzi, M Fathy, Analytical model for connectivity in vehicular ad hoc networks. IEEE Trans Veh Technol. 57(6), 3341-3356 (2008)

8. WL Tan, WC Lau, O Yue, Modeling resource sharing for a road-side access point supporting Drive-thru Internet, in ACM VANET'09, Beijing, China, pp. 33-42 (2009)

9. R Bruno, M Conti, Throughput and fairness analysis of 802.11-based vehicleto-infrastructure data transfers, in IEEE MASS 2011, Valencia, Spain, pp. 232-241 (2011)

10. J An, X Guo, Y Yang, Analysis of collision probability in vehicular ad hoc networks, in ACM GEC'09, Shanghai, China, ACM, New York, pp. 791-794 (2009)

11. TY Wu, S Guizani, WT Lee, KH Liao, Improving RSU service time by distributed sorting mechanism. in Ad Hoc Networks (2010)

12. TH Luan, $X$ Ling, XS Shen, MAC in motion: impact of mobility on the MAC of Drive-thru Internet. IEEE Trans Mobile Comput (2011) http://ieeexplore. ieee.org/stamp/stamp.jsp?tp=\&arnumber=5714694. in press

13. IEEE Trial-Use Standard for Wireless Access in Vehicular Environments (WAVE)-Multi-channel Operation (New York, NY, 2007) IEEE Std 1609.4, IEEE

14. JY Lee, HS Lee, A performance analysis model for IEEE 802.11e EDCA under saturation condition. IEEE Trans Commun. 57(1), 56-63 (2009)

15. H Takagi, Queueing Analysis Vacation and Priority Systems, vol. 1. (NorthHolland, Amsterdam, 1991)

16. Maple 13, (Maplesoft, a division of Waterloo Maple, Inc., Waterloo, ON, 2009)

doi:10.1186/1687-1499-2011-174

Cite this article as: Öztürk et al:: Reaching spatial or networking saturation in VANET. EURASIP Journal on Wireless Communications and Networking 2011 2011:174.

\section{Submit your manuscript to a SpringerOpen ${ }^{\circ}$ journal and benefit from:}

- Convenient online submission

- Rigorous peer review

- Immediate publication on acceptance

- Open access: articles freely available online

- High visibility within the field

- Retaining the copyright to your article

Submit your next manuscript at $\boldsymbol{s p r i n g e r o p e n . c o m ~}$ 